\title{
Survey Paper on Door Level Security using Face Recognition
}

\author{
Harshada B. More ${ }^{1}$, Anjali R. Bodkhe ${ }^{2}$ \\ Department of Comp Science \& Engg, Government College of Engg, Jalgaon Maharashtra, India ${ }^{1,2}$
}

\begin{abstract}
This paper is a review of existing face detection and recognition which is conducted to investigate the result of different approaches in terms of recognition accuracy and some of them are discussed for minimizing processing time point of view. The goal of this paper is to present a critical survey of existing literature on human face recognition. This paper is having two level security which makes the security stronger and reliable. In turn, this give the user more control. In past days, the research is gone on various door lock security systems like traditional security systems which provide indication using alarms. Due to the advancement in recent techniques, some door lock security systems are based on PROTEUS, GSM, GPS, many sensors, iris recognition, software like MATLAB, microcontroller, biometrics like face recognition, face detection, RFID and smart cards. This paper is basically comprised of three subsystems Face detection, Face recognition and automatic door access control.
\end{abstract}

Keywords: Face recognition, detection, PCA, MATLAB, biomatrics, GSM, GPS.

\section{INTRODUCTION}

Nowadays, biometrics data has become popular for using face detection and recognition. This system uses automatic personal identification in access control instead sensor, namely passive infrared receiver (PIR) which of using cards, passwords or pattern. Most of the detects presence of human in front of door, Vibration biometrics data have to be collected by using special sensors for detecting vibrations at door or window area, hardware such as fingerprint scanner, DNA analyzer, and Microcontroller 89E51RD2 for sending signals to palm print scanner. And, the target objects have to touch computer, Camera for capturing images, GSM module for with the required hardware in the stage of data collection. sending messages and a buzzer for alerting authorized The influence of this system is that face recognition does persons.

not require to be touched with any hard-ware. Face is detected significantly by using face detection technique In the field of face recognition, the measurement of the and the entire face recognition is completed without facial images is very high and require considerable amount touching with any hardware. It is one of the few of computing time for classification. The classification and biometrics methods that acquires the qualities of both low intrusiveness and high accuracy. Face recognition technology has range of applications in law enforcement and inspections, access control, information security, smart cards, others. It has many potential applications such as surveillance, credit cards, password security, etc.

So in this paper we proposed a system with two level security checks. This system uses password with face recognition technique for theft detection. First system acquires password from user. If that password matched with authorized password, then current captured image will get compared with authorized image stored along with that password. This two level security reduces the number of comparisons required to match the image with authorized person. If any unauthorized person tries to get access of area, then system raises alarm and sends message to registered mobile number using GSM (Global System for Mobile Communication) module. If any unauthorized person tries to harm the system, vibration sensor sends signal to microcontroller 89E51RD2 which in turns raises alarm. This system helps users for improvement of the door security of sensitive locations by subsequent recognition time can be reduced by reducing dimension of the image data. Principal component analysis (PCA) is one of the famous methods used for feature extraction and data representation.

It not only reduces the dimensionality of the image, but also retains some of the fluctuations in the image data and provides a compact representation of a face image. The key idea of the PCA method is to reconstruct the face images into a small set of attribute feature images, reffered eigenfaces, which are the principal components of the initial training set of the face images. PCA yields projection directions that maximize the total scatter across all classes, i.e., across all face images.

\section{LITERATURE SURVEY}

Door lock security systems are classified based on technology used 1) GSM based, 2) smart card based, 3) Password based, 4) Biometric based, 5) RFID based, 6) Door phone based, 7) Bluetooth based, 8) Social networking sites based, 9) OTP based, 10) Motion detector based, 11) VB based, 12) Combined system. 


\subsection{Password Based Systems}

The programmable electronic code lock device [1] is programmed in such a way that it will operates only with the correct entry of predefined digits. It is also called an integrated combinational type lock. The programmable code lock is shown in Fig 1 as below.

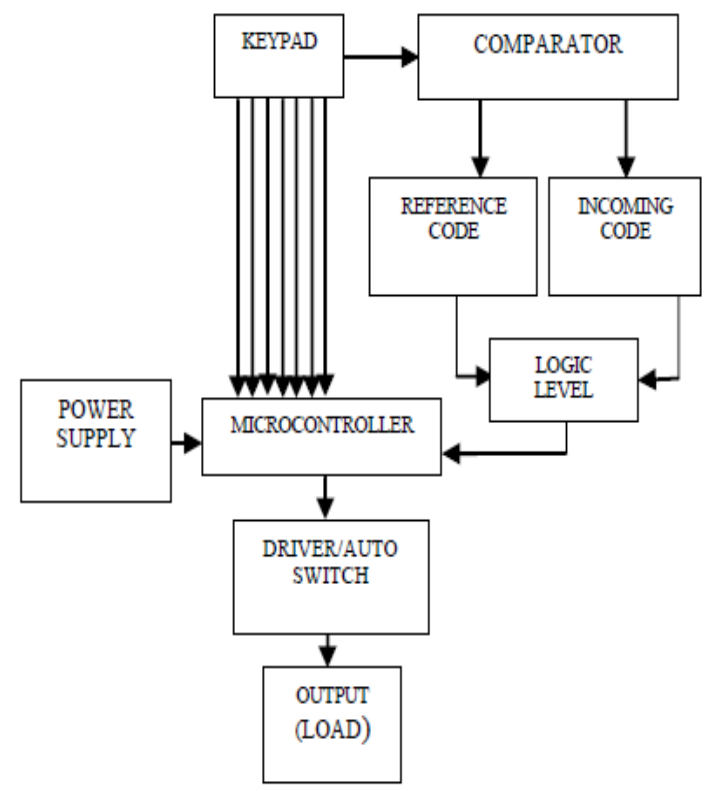

Fig 1: Programmable Electronic Code Lock

Electronics safe is its example. Based on the programmable electronic code lock, the reprogrammable digital door locks [2] were invented in that the password can change any time as it stored in PROM. For operating the device, GSM/CDMA module can be used. When any person calls up from his phone, the call will be received by the system. And the door will opens only if the call is from specified user.

A cellphone controlled password protected door lock system [3] is as shown in Fig 2 which was proposed to open the door with the help of cell phone device by entering a specific code. The user can make a call to a system"s number. This call is responsible for opening or closing of the entry with the use of correct password.

In latest password based system, a more advanced system [4] develops which communicates the owner of the office or house, when any unauthorized person tries to open the code, by giving correct code as well. While closing the door of office/home, the owner has to press the 0 key available on the hex keypad and free the system. The system developed by Annie P. Oommen et. al. [5] allows for changing the password. To open the lock, the entered password must matches with the changed one. In some systems the security dial-up enables through the GSM modem [6], when the unauthorized person enters an invalid password then the controller informs to the owner through GSM modem. Latest security system [7] is designed where the locking security system can be enhanced with the help of GSM and RF wireless technology by using a 4 digit password which provides the authentication.

\subsection{Biometric Based System}

The palmtop recognition is the next step for fingerprint recognition. It [8] operates on the image of palmtop. Firstly system takes an image of the palmtop then it works on that image by partitioning it and process is required. At the end, verify the right person. Hence, it reduces the chances of error in other human recognition methods and clarifies the problems which were faced in the fingerprint recognition. The biometric technique is very useful in bank lockers. Except fingerprint recognition the vein detector and iris scanner gives best and accurate result so, in the bank security system [9], microcontroller continuously observes the Vein Detector and Iris Scanner through keypad authenticated codes. During night the wireless motion detector will be active, if any variation occurs in its output, it will be sensed by the controller and alert sounds will be given by it.

Recently, the fast based principal component analysis approach is proposed in which the modification of principal component analysis approach for the face recognition and face detection process is done [10]. The image is captured by the web camera and it gets matched with the image stored in the database. New advanced door lock security systems are available based on the pattern of the human iris for providing a high level of security. And to make the system more efficient $\mathrm{n}$ reliable the duplication is done in MATLAB [1].

\subsection{GSM Based Systems}

In many door lock security systems, GSM is used for communication purpose. The purpose of a work cultivated by utilization of a circuits like a GSM module which gets activated by a controller [2] for sending SMS in emergency to proprietor and for sending corresponding services of security at the time of break in. For detecting obstacles, the system requires various sensors. It gathers data from the sensors and settles on a choice. With the help of GSM module, sends SMS to a respective number. A recently created model for security of door [3] easily controlled like remote control operations by a GSM hand set acts as the transmitter and the other GSM phone set with the DTMF associated with the motor attached to door with the use of DTMF decoder, a stepper motor and microcontroller unit.

Nowadays people want to be secure though they are away from home so, the work proposed by Jayashri Bangali et. al.[4]. When the owner is not at his home, security of home and important things is the big issue in front of all. Two frameworks were created which depends on GSM based technology. For detection of the gate-crashes, it takes place by capturing image through web camera. When peoples are not at their homes, the system sends notification in terms of SMS to the crisis number. A novel administrator based system [5] can login without any stretch to the system and can see guests record and listen 
their recorded messages and also automatically lock the door using mobile communication technology.

\section{FACE RECOGNITION SYSTEM}

A pattern recognition task performed exclusively on faces is termed as face recognition. It can be described as classifying a face either known or unknown, after matching it with stored known individuals as a database. It is also advantageous to have a system that has the capability of learning to identify unknown faces. The outline of typical face recognition system implemented specifically for N-PCA is given in Figure1. There are five main functional blocks, whose responsibilities are as below.

A. The acquisition module This is the entry point of the face recognition process. The user gives the face image as the input to face recognition system in this module.

B. The pre-processing module In this module the images are normalized to improve the recognition of the system. The pre-processing steps implemented are as follows: - Image size normalization - Background removal - Translation and rotational normalizations • Illumination normalization

C. The feature extraction module After the preprocessing the normalized face image is given as input to the feature extraction module to find the key features that will be used for classification. The module composes a feature vector that is well enough to represent the face image.

D. The classification module With the help of a pattern classifier, the extracted features of face image are compared with the ones stored in the face database. The It is used to match the test face image is then classified as either known or unknown.

E. Face database image with the train images stored in a database. If the face is recognized as "unknown", face images can then be added to the database for further comparisons.

\section{FACE RECOGNITION ALGORITHM}

Face recognition systems are now providing the need for security to cope up with the current misdeeds. It is really influential with the market information that undoubtedly depicts the rising fascination of the face recognition system. In the present era, the threat of protecting the information or physical property is becoming more and more difficult and important. Now a day the crimes of computer hackings, credit card fraud or security violation in a company or government building has noticed to be increased [4]. The face recognition system consists of two important steps, the feature extraction and the classification. Face recognition has a challenge to perform in real time. Raw face image may consume a long time to recognize since it go through from a huge amount of pixels. One needs to reduce the amounts of pixels. This is called dimensionality reduction or feature extraction, to save time for the decision step. Feature extraction refers to convert face space into a feature space. In the feature space, the face database is represented by a reduced number of features that retain most of the important information of the original faces. The most popular method to achieve this target is through applying the Eigenfaces algorithm [9]. The Eigenfaces algorithm is a classical statistical method by using the linear KarhumenLoeve transformation (KLT) also known as Principal component analysis. The PCA calculates the eigenvectors of the covariance matrix of the input face space. These eigenvectors define a new face space where the images are represented. In contrast to linear PCA, N-PCA has been developed.

\subsection{Principal Component Analysis}

On the field of face Detection most of the common methodsemploy Principal Component Analysis. Principal ComponentAnalysis is based on the Karhunen-Loeve (KL), or HostellingTransform, which is the optimal linear method for[9] reducingredundancy, in the least mean squared reconstruction errorsense. 1. PCA became popular for face Detection with thesuccess of eigenfaces. The idea of principal component analysis is based on the introduction of linear transformation of the co-ordinates of asystem. "The three axes of the new co-ordinate systemcoincide with the directions of the three largest dispertion of thepoint distributions."In the new co-ordinate system that we have now the data isuncorrected with the data we had in the first co-ordinatesystem. [2]For face Detection, given dataset of $\mathrm{N}$ training images, wecreate $\mathrm{N}$ d-dimensional vectors, where each pixel is a uniquedimension. The principal components of this set of vectors iscomputed in order to obtain a $\mathrm{d} x \mathrm{~m}$ projection matrix, W.comparitively the original image where $\mu$ is the mean, of thexi and the reconstruction is perfect when $\mathrm{m}=$ d.For the comparison we are going to use two different PCAalgorithms. The first algorithm is and storingthe weight of vectors for each person's image in the trainingset, so the actual training data is not necessary. In the secondalgorithm each weight of each image is stored individually, isa memory-based algorithm. For that we need more storingspace but the performance is better.In order to implement the Principal component analysis inMATLAB we simply have to use the command prepare.

Thesyntax of the command is

ptrans, transMat $=\operatorname{prepca}\left(\mathrm{P}, \mathrm{min} \_\right.$frac $)$

Prepca pre-processes the network input training set byapplying a principal component analysis. This analysistransforms the input data so that the elements of the inputvector set will be, which contribute more than a specified fraction(min_frac) of the total deviation in the data set.

Prepca uncorrected. In addition, the size of the input vectors may be reduced by retaining[10] only those components takes these inputs the matrix of centred input(column) vectors, the minimum fraction deviation 
componentto keep and as result returns the transformed data set and thetransformation matrix.

\section{A. Algorithm}

Principal component analysis uses singular value decomposition to compute the principal components. A matrix whose rows consist of the eigenvectors of the input covariance matrix multiplies the input vectors. This produces transformed input vectors whose components are uncorrected and ordered according to the magnitude of their variance. Those components, which contribute only a small amount to the total diversion in the data set, are eliminated. It is assumed that the input data set has already been normalised sothat it has a zero mean. In our test we are going to use two different "versions' of PCA. In the first one the centroid of the weight vectors for each person's images in the training set is computed and stored. On the other hand in PCA-2 a memory based variant of PCA, each of the weight vectors in individually computed and stored.

\section{B. Eigenfaces}

Human face Detection is a very difficult and practicalproblem in the field of pattern Detection. On thefoundation ofanalysis of the present methods on human face Detection,[12]a new technique of image feature extraction is presented.And bind with the artificial neural network, a newmethod on human face Detection is brought up. By extractionthe sample pattern's algebraic feature, the human face image'seigenvalues, the neural network classifier is trained forDetection. The Kohonen network we adopted can adaptivelymodify its bottom up weights in the course of learning.Experimental results show that this method not only utilisesthe feature aspect of eigenvalues but also has the learningability of neural network. It has better discriminate ability compared with the nearest classifier. The method this paperfocused on has wide application area. The adaptive neuralnetwork classifier can be used in other tasks of patternDetection.In order to calculate the eigenfaces and eigenvalues inMATLAB we generally to use the command eig. The syntax of thecommand is

$\mathrm{d}=\operatorname{eig}(\mathrm{A})$

$\mathrm{V}, \mathrm{D}=\operatorname{eig}(\mathrm{A})$

$\mathrm{V}, \mathrm{D}=\operatorname{eig}(\mathrm{A}$, 'nobalance')

$\mathrm{d}=\operatorname{eig}(\mathrm{A}, \mathrm{B})$

$\mathrm{V}, \mathrm{D}=\operatorname{eig}(\mathrm{A}, \mathrm{B})$

$d=\operatorname{eig}(A)$ returns a vector of the eigenvalues of matrix $A$.

$\mathrm{V}, \mathrm{D}=\operatorname{eig}(\mathrm{A})$ genarates matrices of eigenvalues (D) and eigenvectors $(V)$ of [13] matrix $A$, so that $A * V=V^{*} D$.

MatrixD is the canonical form of $\mathrm{A}$, a diagonal matrix with A'seigenvalues on the main diagonal. Matrix $\mathrm{V}$ is the modalmatrix, its columns are the eigenvectors of $\mathrm{A}$. Theeigenvectors are scaled so that the norm of each is 1.0. Thenwe use $\mathrm{W}, \mathrm{D}=\operatorname{eig}\left(\mathrm{A}^{\prime}\right) ; \mathrm{W}=\mathrm{W}^{\prime}$ in order to compute the lefteigenvectors, which satisfy $\mathrm{W}^{*} \mathrm{~A}=\mathrm{D}^{*} \mathrm{~W} \cdot \mathrm{V}, \mathrm{D}=$ eig(A,'nobalance') finds eigenvalues and eigenvectors without a preliminary balancing step. Ordinarily,balancing improves the situating of the input matrix,enabling more accurate computation of the eigenvectors andeigenvalues. However, if a matrix contains small elements thatare really due to round-off error, balancing may scope them upto make them as significant as the other elements of theoriginal matrix, leading to incorrect eigenvectors. We can usethe no balance option in this event.d $=\operatorname{eig}(A, B)$ returns a vector containing the generalisedeigenvalues, if $\mathrm{A}$ and $\mathrm{B}$ are square matrices. $\mathrm{V}, \mathrm{D}=\operatorname{eig}(\mathrm{A}, \mathrm{B})$ produces $\mathrm{a}$ diagonal matrix $\mathrm{D}$ of generalised eigenvalues and afull matrix $\mathrm{V}$ whose columns are the corresponding eigen vectors so that $\mathrm{A} * \mathrm{~V}=\mathrm{B} * \mathrm{~V} * \mathrm{D}$. The eigenvectors are scaled so that the norm of each is 1.0 .

\section{Euclidean distance}

One of the trick on which face Detection is based is thedistance measures, between to points. The problem of findingthe distance between two or more point of a set is defined asthe Euclidean distance. The Euclidean distance is usuallyreferred to the closest distance between two or more points

\section{COMPONENTS OF FACE RECOGNITION SYSTEMS}

The Face Recognition system includes:

An automated mechanism that scans and captures a digital or an analog image /of living personal characteristics (Enrollment module).

$\square$ Another entity which handles compression, processing, storage and compression of the captured data with stored data (Database).

The third interfaces with the application system (Identification module).

The different Modules of Face Recognition System as shown in the Figure. 2 are:

\section{A. User Interface:}

User interface captures the analog or digital image of the person's face. In this we take number of photos of a single person either in analog or in a digital form.

\section{B. Enrollment Module:}

In the enrollment module the obtained sample is preprocessed and analyzed. This analyzed data is stored in the database for the purpose of future comparison.

\section{Database:}

The database compresses the obtained sample and stores it properly. It should have retrieval property also so that it compares all the stored sample with the newly obtained sample and retrieves the matched sample for the purpose of verification of the user and determine whether the match declared is right or wrong.

\section{Verification:}

The verification module also consists of a pre-processing system. Verification means the system checks as to who the person says he or she is and gives a yes or no decision. In this module the newly obtained sample is compared and pre-processed with the sample stored in the database. The 
decisionis taken depending on the match obtained from the database.Appropriately the sample is accepted or rejected. Instead ofverification module we can make use of identification module.For each comparison made a match score is given. Thedecision to accept or reject the sample depends on this matchscore falling above or below a preordenaid threshold.

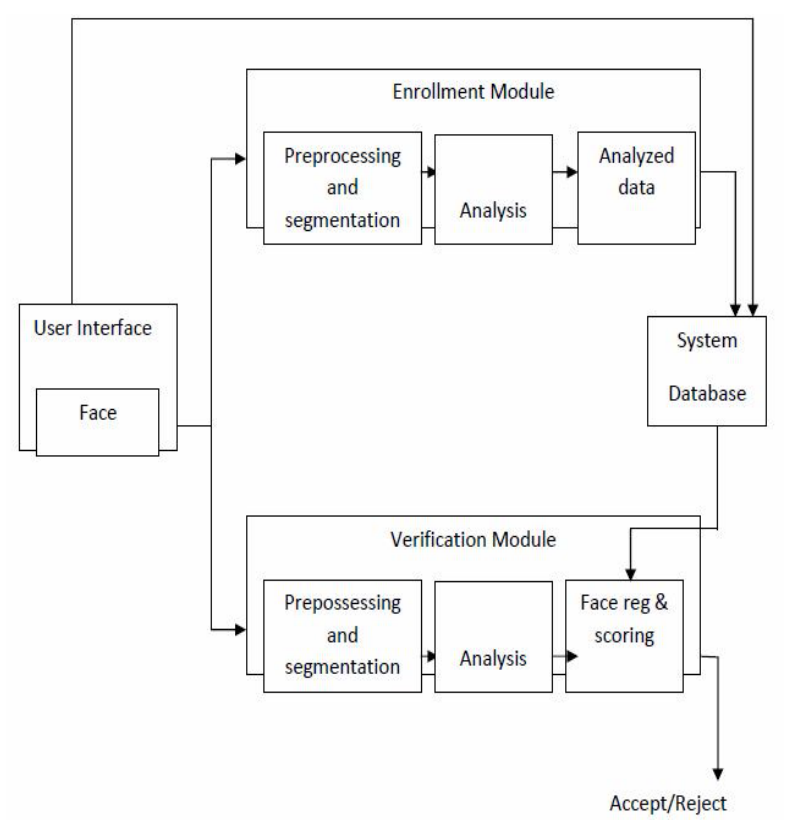

\section{DISCUSSIONS AND CONCLUSIONS}

In this paper, automatic door access system using face recognition and detection is presented. Automatic face detection and recognition is done by java program on PC. Microcontroller is used to control the door access system. Door is opened immediately after confirming that the person is authenticated. After 2 seconds, door is closed automatically. In real time, 2 seconds are not enough time to enter a person. So, longer time should be set for realtime condition. For face recognition, Principal Component Analysis(PCA) method is used to extract the important features of facial images. Therefore, PCA method reduces the dimension of the dataset, this system can detect and recognize an image within one second. Therefore, this system can be used in automatic verification of people to improve door security for outsiders without needing security guards and wasting too many time.

\section{REFERENCES}

[1] Mohammad Amanullah "MICROCONTROLLER BASED REPROGRAMMABLE DIGITAL DOOR LOCK SECURITY SYSTEM BY USING KEYPAD \& GSM/CDMA TECHNOLOGY", IOSR Journal of Electrical and Electronics Engineering (IOSR - JEEE), Volume 4, Issue 6 (Mar. - Apr. 2013).

[2] Kawser Wazed Nafi, Tonny Shekha Kar, Sayed Anisul Hoque, “ AN ADVANCED DOOR LOCK SECURITY SYSTEM USING PALMTOP RECOGNITION SYSTEM", International Journal of Computer Applications (0975 - 8887), Volume 56- No.17, October 2012
[3] W. Zhao, R. Chellappa, P. Phillips, A. Rosenfeld, "Face recognition: a literature survey", ACM Computing Surveys vol. 35, pp.399-458, December 2003.

[4] M. Turk and A. Pentland, "Eigenfaces for Recognition," J. Cognitive Neuroscience, vol. 3, no. 1, pp. 71-86, 1991.

[5] Pantic M. and Rothkrantz L.J.M., "Automatic analysis of facial expressions: the state of the art ", IEEE Trans. Pattern Analysis and Machine Intelligence, Vol. 22, No. 12, pp. 1424-1445, 2000.

[6] "Analysis of PCA-Based and Fisher Discriminant-Based Image

[7] Recognition Algorithms:” Wendy S. Yambor, July 2000 (Technical Report CS-00-103, Computer Science).

[8] "Survey: Face recognition systems:" C. Bunney, Biometric Technology Today, Vol. 5, No. 4, pp. 8-12, 1997.

[9] "Research on Face Recognition Based on PCA:" Hong Duan1, Ruohe

[10] Yan1, Kunhui Lin, 2008 International Seminar on Future Information Technology and Management Engineering

[11] Moon H, Phillips P J, "Computational and performance aspects of PCA-based face-recognition algorithms", Perception 30(3) PP.303

[12] 321,2001

[13] Shah J., Sharif M., Raza M. and Azeem A., A Survey: Linear and Nonlinear PCA Based Face Recognition 